\title{
Priority Aware MAC Protocol for Delay-Bounded Applications in Wireless Sensor Networks
}

\author{
Hye Yun Kim ${ }^{1}$, Seong Cheol Kim ${ }^{2 *}$ and Hyun Joo Park \\ ${ }^{1}$ Department of System Semiconductor Engineering \\ Sangmyung University, Cheonan, Korea \\ ${ }^{2 *}$ Department of Convergence Electronics Engineering \\ Sangmyung University, Seoul, Korea \\ ${ }^{3}$ Department of Smart Information and Telecommunication Engineering \\ Sangmyung University, Cheonan, Korea \\ ${ }^{1}$ hykim@smu.ac.kr, ${ }^{2}$ sckim@smu.ac.kr, ${ }^{3}$ cathy2369@smu.ac.kr
}

\begin{abstract}
In this paper, we presented a priority aware MAC Protocol for delay-bounded applications, which is called PA-MAC. The PA-MAC protocol was designed for some applications such as field monitoring in which both abnormal data and normal data may be collected. In this kind of WSNs applications, sensor nodes may need to send both abnormal data and normal data. Abnormal data packet may be generated when there is an event such as exceeding threshold value or object appearance. These events are generally critical, so the collected abnormal data should be transmitted in limited time to their final destination node faster than the normal data. Therefore, the priority packet transmission mechanism is necessary in these applications. Experiment results show that the PA-MAC protocol reduces entire packet transmission delay and average node energy consumption in comparison to existing related MAC protocols.
\end{abstract}

Keywords: Wireless Sensor Networks, Quality of Service (QoS), Priority, Multiple Access Control (MAC), delay

\section{Introduction}

Wireless sensor networks (WSNs) have been widely used in many applications such as those in the field of environment monitoring, health monitoring, and military surveillance. Wireless sensor networks (WSNs) consist of a large number of wireless battery-operated sensor nodes capable of sensing or measuring the environments. It is generally difficult to replace or recharge the batteries used in WSNs. To prolong the network lifetime, it is important to solve the problem on energy-efficiency in the design of WSNs [1-6]. The Medium Access Control (MAC) layer is basically responsible for the setup of communication connections between sensor nodes. The MAC protocol controls the access to the shared wireless channel by several sensor nodes. The primary goal of WSNs is energy efficiency due to the limited energy of sensor battery [7-9]. Idle listening state is a major source of energy consumption of the sensor node. A sensor node stays in ON mode with radio turned $\mathrm{ON}$ in idle listening to capture incoming packets. To reduce the idle listening energy consumption, many MAC protocol design approaches have been suggested. Duty cycling is one of the solutions to this problem [10-12]. In duty cycling scheme, sensor nodes periodically stay between ON and OFF modes. When in ON mode, a node can transmit or receive data packet, whereas when in OFF mode, a node turns its

Received (May 12, 2018), Review Result (July 21, 2018), Accepted (September 6, 2018)

* Corresponding Author 
power off to save energy. A duty cycle MAC protocol can also be divided into two groups: synchronous and asynchronous. Using synchronous MAC protocol, nodes share the scheduling information with each other. The scheduling information specifies the active and sleep periods by exchanging special control packets to each other during common active state. The main drawback of this protocol is the complexity caused by the synchronization of the active period information. A number of research works to reduce transmission delay in synchronized duty cycle MAC protocols for WSNs have been suggested [9-11]. On the other hand, each node operates independently in an asynchronous MAC protocol. In an asynchronous MAC protocol, each node uses its own duty cycle schedule. Exchanging of synchronization information between nodes is not required in an asynchronous protocol. Instead of exchanging synchronization information, the nodes in asynchronous protocol use preamble transmission. So, an asynchronous protocol is only adequate for light traffic-load environment because of the preamble transmissions overhead. In the event-driven applications of Wireless Sensor Networks (WSNs) such as object appearance, wild animal monitoring, and fire monitoring, burst data may be generated when such an event occurs. For example, in an object tracking application, a node needs to collect information continuously when an object appears. As the object moves, the sensor node must continue to collect information and send the collected information to the sink node. Furthermore, the measured data should be sent to the sink node in a predefined time. Even so, normal measured data may be generated when no event occurs. As existing approaches are mainly optimized for energy consumption of the nodes, we found that nodes become less efficient in transmission delay as burst data is generated. In this paper, we propose a PA-MAC protocol that can be applied well to such a network environment. The rest of this paper is organized as follows: Section 2 discusses the works related to the proposed PA-MAC; Section 3 presents the details of the design of the proposed priority and delay aware packet transmission MAC Protocol; Section 4 presents the results of the performance evaluation of the PA-MAC; and Section 5 highlights the conclusions of this research.

\section{Related Works}

MAC protocols for WSNs can be divided into two parts: contention-based and contention-free protocols. In a contention-based protocol, sensor nodes should stay in ON state to receive possible incoming packets. So nodes in this scheme consume much energy due to the long period of idle listening. So many research works are focusing on saving energy of node by turning the radio off as soon as possible. On the other hand, contentionfree protocols try to detect the neighbor nodes before allocating collision-free channels to a link. To reduce packet transmission delay in synchronized duty cycle MAC protocol for WSNs, a number of approaches have been suggested. S-MAC [1], T-MAC [3], RMAC [10], DW-MAC [11], and SR-MAC [12] are the examples of synchronous approaches. RMAC [10] was designed to reduce packet delivery latency in multi-hop network environment. In RMAC, a special control frame PION (Pioneer frame) is used to forward packets over multiple hops efficiently. The PION frame works as RTS/CTS and ACK. If a node wants to send data packet, the node needs to send a PION frame first. Sending a PION frame is to request packet communication. The PION frame from sender node is forwarded over multiple hops during a Data period in order to inform other nodes. And during a Sleep period, all the other nodes stay in sleep mode except for some nodes that has sent and relayed a PION frame. The one of drawbacks of the RMAC is that all sender nodes always try to send their packets at the beginning of a Sleep period. In other words, all nodes that sent PION frames to their neighbor nodes, may send their data packet at the beginning of Sleep period. So packet collisions at the beginning of the Sleep period may occur if more than two nodes sent PION frames during a Data period. To solve this problem, DW-MAC (Demand Wakeup MAC) was proposed. DW-MAC is also a 
synchronized duty cycle MAC protocol. In DW-MAC [11], each operational cycle is also divided into three periods like RMAC: Sync, Data, and Sleep period. But DW-MAC uses scheduling frame $(\mathrm{SCH})$ as a control frame. The nodes in DW-MAC wake up on demand during the Sleep period in order to receive or transmit a packet. DW-MAC uses one-toone mapping function between a Data period and a Sleep period to prevent packets collision. As shown in the Figure 1, node A tries to send a data packet to node B. Node A first sends its $\mathrm{SCH}$ frame $\mathrm{T}_{1}$ time units at the start of the Data period. The frame transmission time is $\mathrm{T}_{3}$ From the mapping function Eq. (1), node A then can send its data packet $T_{2}$ at the start of following Sleep period. Here $T_{4}$ denotes for a maximum wakeup time duration satisfying equation (1).

$$
\frac{T_{2}}{T_{1}}=\frac{T_{4}}{T_{3}}=\frac{T_{\text {Sleep }}}{T_{\text {Data }}}
$$

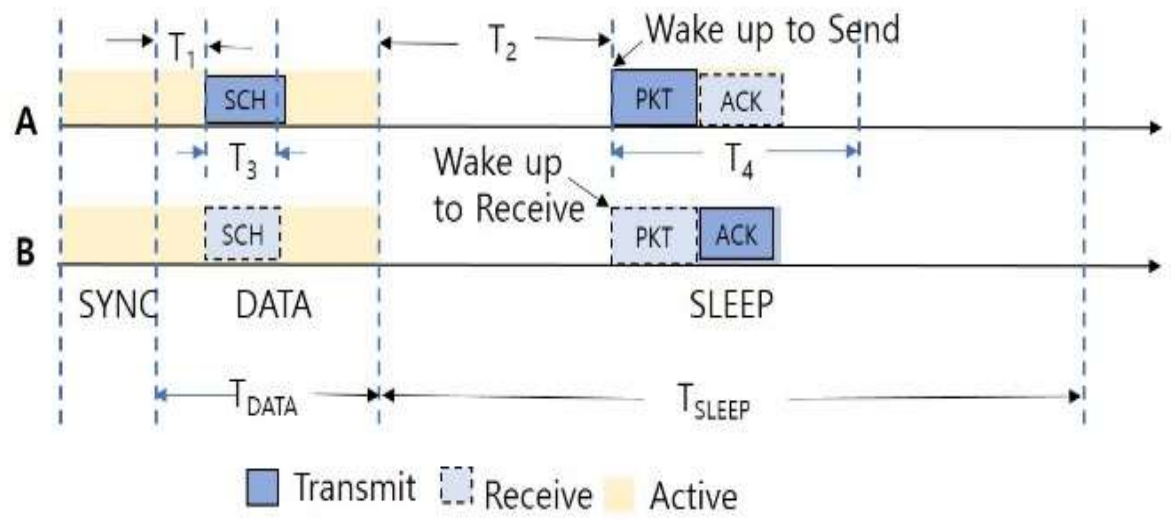

Figure 1. Packet Transmission Scheduling in DW-MAC

Using the mapping function between a Data period and the following Sleep period, DW-MAC resolves the packet collision problem experienced in RMAC. But the DWMAC protocol allows nodes to send only one packet during a Sleep period. In case of event-driven application, in which multiple packets may be generated by a single node, more operational cycles may be needed by a node to transmit all the generated packets in DW-MAC. To transmit burst data packets, several operational cycles are required. So multiple packets transmission delay may significantly increase. To resolve this problem, SR-MAC [12] was suggested. SR-MAC is also a synchronous duty cycling MAC protocol. Each operational cycle in SR-MAC is also divided into three parts: Sync, Data, and Sleep periods. To synchronize the clock of neighbor nodes, Sync period is used in SR-MAC. The SR-MAC uses a scheduling mechanism that reserves few time slots in one Sleep period. It allows the nodes to transmit multiple data packet in an operational cycle. In SR-MAC, Sleep period and Data period have the same slot number. For transmitting data packets in SR-MAC, the node sends its SRF (slot-reserved frame) to its neighbor nodes. The SRF frame is a special control frame such as PION in RMAC or SCH in DWMAC. The SRF frame plays as a handshake frame like RTS/CTS and is used for slot reservation. The sender node sends SRF frame during Data period to serve sleep slot for packet transmission in Sleep period. The packet scheduling based on slot reservation mechanism in SR-MAC is shown in the Figure 2. The node $\mathrm{S}$ wants to send data packet to node $\mathrm{R}$. Then the node $\mathrm{S}$ first contends the channel access with other nodes in the cluster. After getting channel access, the node $S$ transmits its SRF frame to reserve slot in the Sleep period. So nodes S and R both reserve same slot, $k_{t h}$ slot in the Sleep period as shown in the Figure 2. Using the slot reservation mechanism, packets transmission in Sleep period can be succeeded without collision. Furthermore, multiple packets on a 
single node can be transmitted in one operational cycle through multiple slots reservation. For multiple packets transmission in a single cycle, a Sleep period is divided into several frames. All frames have the same number of slots and node can send multiple packets at the same position of slot in every frame. Between the number of slot in a frame and the slot time is shown in the following Eq. (2).

$$
T_{\text {sleep slot }} \leq \frac{T_{\text {Sleep }}}{M}
$$

Where $T_{\text {sleep slot }}$ is the length of a slot, $M$ is a number of slots, and $T_{\text {Sleep }}$ is the length of Sleep period. But SR-MAC faces the problem that the number of multiple packets on a single node may be limited by the fixed length of Sleep period.

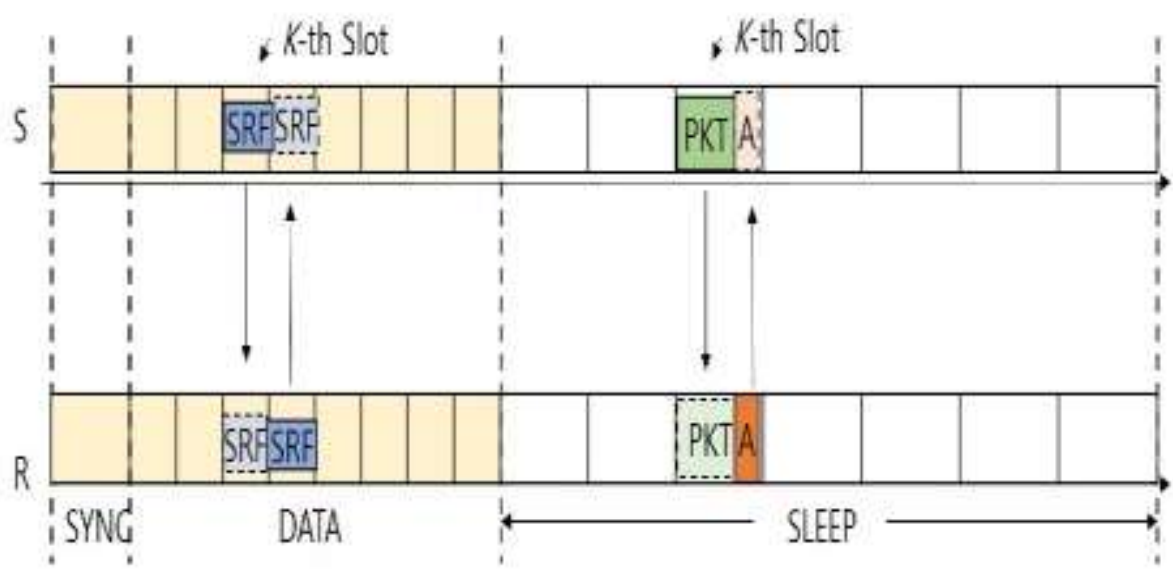

\section{Figure 2. Packet Transmission Scheduling with Slot-Reservation in DW- MAC}

In an event detection application such as object tracking and military surveillance, burst data may be generated when an event occurs. Therefore, many data packets should be transmitted in a predefined time to the sink node. To cope with this problem, a delay efficient and burst traffic friendly scheme is required [13-15]. To solve this problem, BTF-MAC (Bursty Traffics Friendly MAC) was proposed. The BTF-MAC [15] is also a synchronous duty-cycle MAC protocol. The BTF-MAC uses a slot-reserved mechanism like SR-MAC and an operational period extension mechanism according to the traffics is used. The BTF-MAC protocol uses duty cycling scheme which is a widely used scheme in wireless sensor networks to reduce energy consumption caused by idle listening. But additional delay in packet transmission is inevitable using this scheme. This duty cycling scheme uses ON and OFF states in every sensor node. During OFF state, a node stays in power off state, in which the node cannot participate in transmitting or receiving data packet. And synchronization between nodes is necessary in order to transmit a data packet from one node to another. At this time both nodes should be in ON state. The BTF-MAC protocol one operation period is divided into 4 parts; Sync1 period, Data period, Sync2 period, and Sleep period. All sensor nodes in BTF-MAC wake up at the beginning of the SYNC1 period and send special control packet periodically to synchronize with their neighbor nodes. During the Data period, sender nodes send control frames to their neighbor nodes. The control frame is SRF. In addition to the sender address, receiver address and duration of the transmission as in RTS/CTS, the SRF includes the number of slots and the number of packets. Figure 3 shows an example of the node's schedule in BPF-MAC scheme Node A has data packet to send. Node A sends its SRF frame during the $2^{\text {nd }}$ slot in Data period. Since the SRF frame is the first frame, node A can deliver its 
data packet at the beginning of each frame. If node A has several number of packets to send, the Sleep period can be expanded. With this operational cycle expansion capability, sensor nodes can send several packets in one operational cycle. With this structure, sink node can send frame expansion information to its neighbor nodes during the Sync2 period. In the Sleep period, packet transmission is carried out between sender node and receiver node. Since only the nodes that receive slot reservation information from the sink node in the Sync2 period will wake up in their allocated slot times, energy waste of nodes in sleep mode can be reduced. But priority packet transmission scheme is not included in the BTF-MAC.

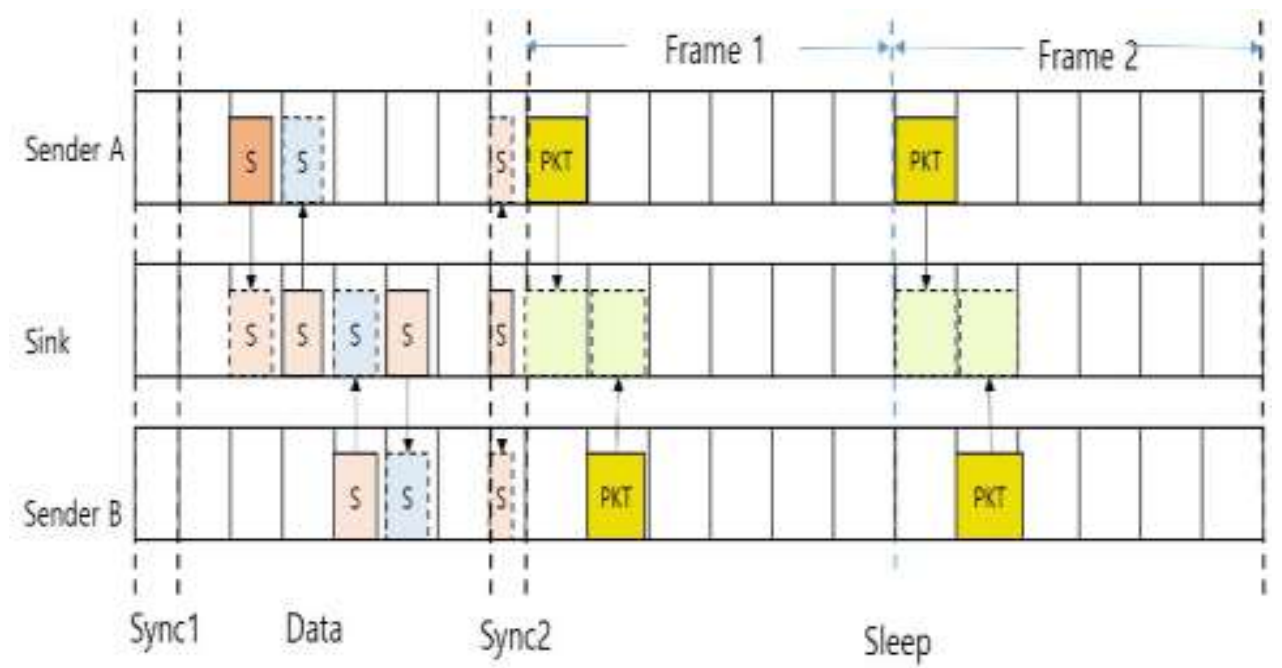

Figure 3. Packet Transmission Scheduling in BTF-MAC

\section{Design of PA-MAC Protocol}

\subsection{Overview}

The Sleep period is also divided into $M$ slots. But the Sleep period can be composed of several frames with $M$ slots. The number of frames in Sleep period depends on the number of packets from sender node. Unlike the existing MAC protocol, PA-MAC adopts a flexible operational cycle according to the data traffic. In other words, the length of Sleep period in PA-MAC is flexible. Any node which sends its SIF frame first in Data period, can send its data packet at the beginning of Sleep period of each frame. All the other nodes except those which have pending packets to send turn their power off and stay in sleep state after Data period. During the Sync2 period, all nodes wake up again to get the slot reservation and the operational cycle expansion information from a sink node. In the proposed scheme, the control frame is the slot-reserved and operational cycle expansion informationinformed frame also called SIF. In addition to the sender address, receiver address and duration of the transmission as in RTS/CTS, the SIF includes the number of slots, number of packets and packet priority information. The node that sends the SIF frame with priority data packet first can send its data packet at the beginning of the Sleep period of each frame. The difference of PA-MAC from BTF-MAC is that the proposed scheme has the function of supporting priority packet transmission and using multiple SIF frames. 


\subsection{Special Period for Priority Data Packets}

As described in the previous section, the sender node that sends the SIF frame first can send its data packet at the beginning of the Sleep period of each frame. So several empty slots may remain at the end of the Data period, as shown in the Figure 4. If a node, whose data is NOT the priority packet, sends an SIF frame first, the packet will be transmitted after the more urgent packet. But if the sink node receives only no-priority packets, then the node with first sent SIF frame can send its data packet at the beginning of Data period. The special period consists of these empty slots in the PA-MAC protocol. Any node that detects an event such as fire or wild animal appearance can send more data packet using these empty slots of the special period. We may consider these urgent data packet as the priority packet. So total transmission delay of these priority packets can be reduced. If only one node sends SIF frame during Data period, then the node can transmit its data packets on the special period without any collision.

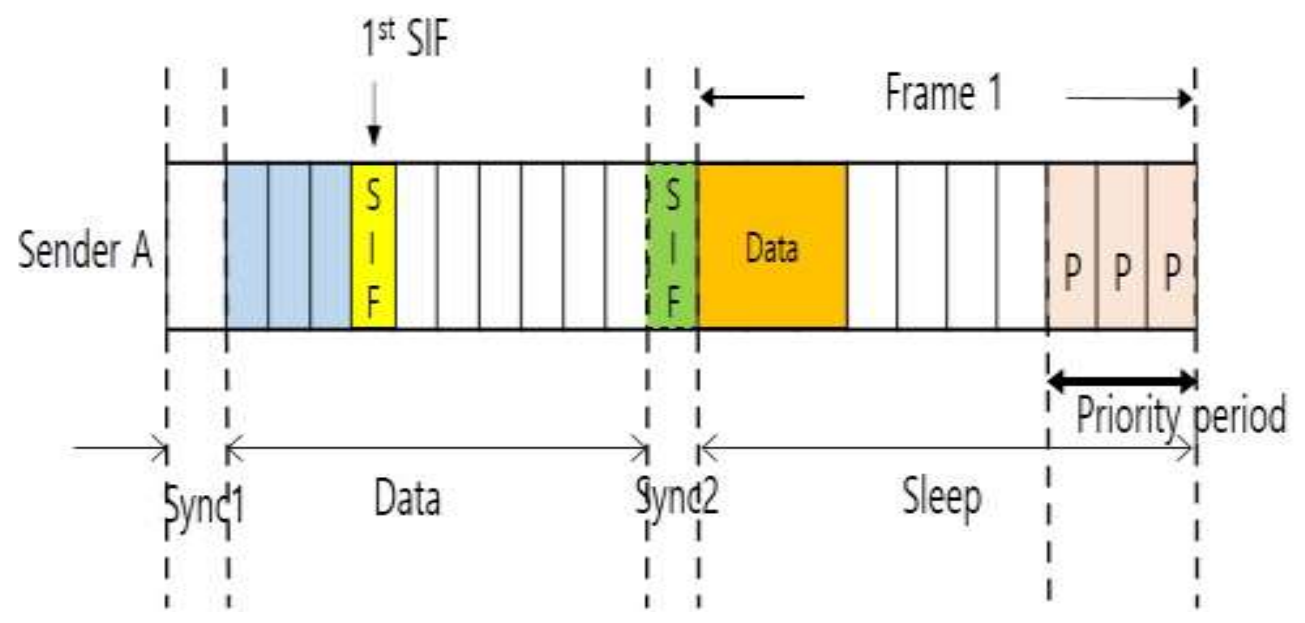

Figure 4. Packet Transmission Scheduling in PA-MAC

In this paper, we present a priority and delay aware packet transmission MAC Protocol which we call PA-MAC.

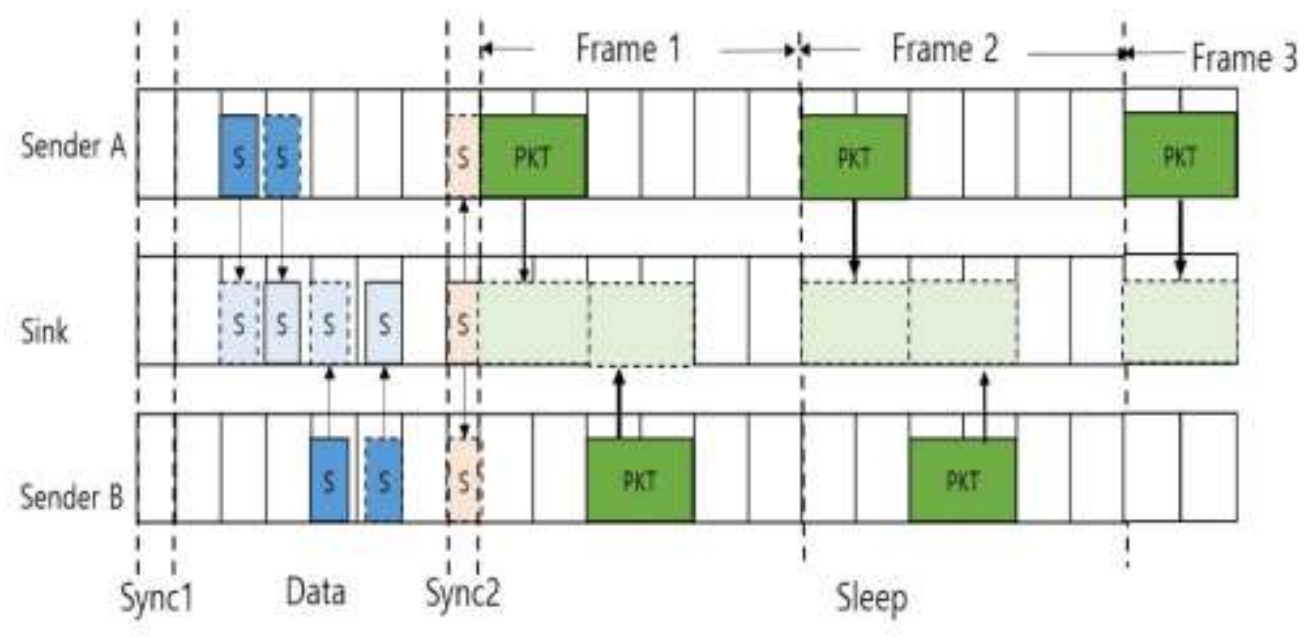

Figure 5. Packet Transmission Scheduling in PA-MAC

An example of packet transmission scheduling in PA-MAC is shown in the Figure 5. 


\section{Performance Evaluation}

Table 1 shows the networking and energy parameters of the sensor node in the proposed scheme, PA-MAC. In the test, the researchers assumed that all sender nodes are located one hop away from the sink node. The network is composed of 39 sensor nodes and one sink node. And all the data packets from sensor nodes are directed to the sink node.

Table 1. Network Parameters

\begin{tabular}{l|l|l|l}
\hline Parameter & Value & Parameter & Value \\
\hline \hline Bandwidth & $20 \mathrm{Kbps}$ & Data pkt size & 50 Bytes \\
\hline$T_{T_{x}}$ power & $0.5 \mathrm{~W}$ & Tsync1 & $55.2 \mathrm{~ms}$ \\
\hline$R_{\bar{x}}$ power & $0.5 \mathrm{~W}$ & Tsync2 & $10 \mathrm{~ms}$ \\
\hline Idle Power & $0.45 \mathrm{~W}$ & $T_{\text {data }}$ & $142.0 \mathrm{~ms}$ \\
\hline Sleep Power & $0.05 \mathrm{~W}$ & Tsleep & $426.0 \mathrm{~ms}$ \\
\hline SIFS & $5 \mathrm{~ms}$ & PION & 14 Bytes \\
\hline DIFS & $10 \mathrm{~ms}$ & SRF, SIF & 14 Bytes \\
\hline RTS,CTS,ACK & $10 \mathrm{Bytes}$ & & \\
\hline
\end{tabular}

The sink node is located at the center of the network structure. Finally, it is assumed that each node contains $50 \%$ priority data packets to transmit. For easy comparison, it is assumed that one frame in a Sleep period is composed of 7 slots and a frame can be extended by 4 in maximum. And it is anticipated that a node could generate three to fourteen data packets when an abnormal condition occurs. The proponents ran ten simulation tests and calculated the mean value of each network performance parameter. Figure 6 shows the average priority packet transmission delay comparison with number of data packet generated from a node. As shown in the Figure 6 the PA-MAC protocol outperforms other related protocols in packet transmission speed between sensor nodes and sink node. For example, under heavy traffic, PA-MAC reduces priority packet transmission delay by more than $150 \%$ on average. Because DW-MAC and SR-MAC cannot transmit all data packet in a single operational cycle when number of packets exceeds 6 , the overall average transmission time increases. This is also due to the fact that in PA-MAC priority packet are always transmitted fast.

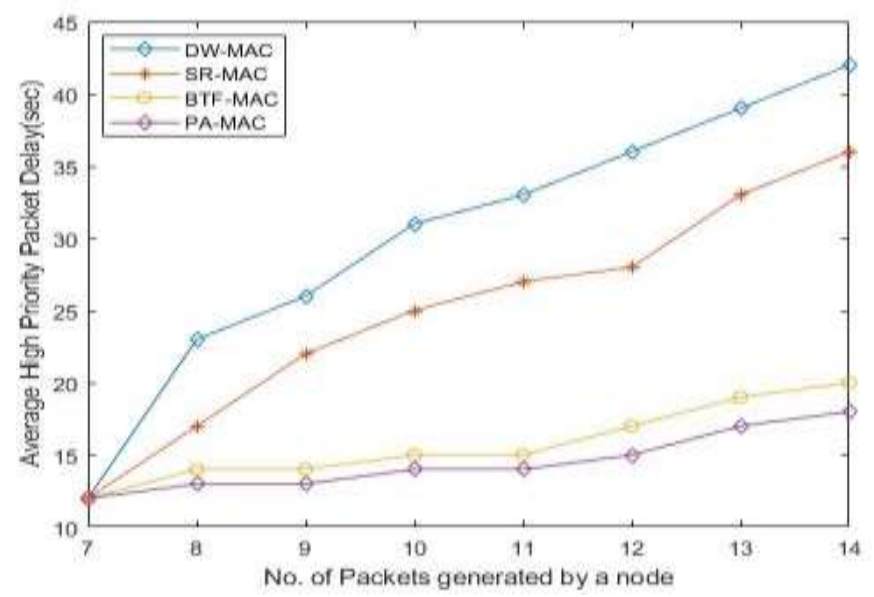

Figure 6. Average High Priority Packet Delay versus No. of packets 


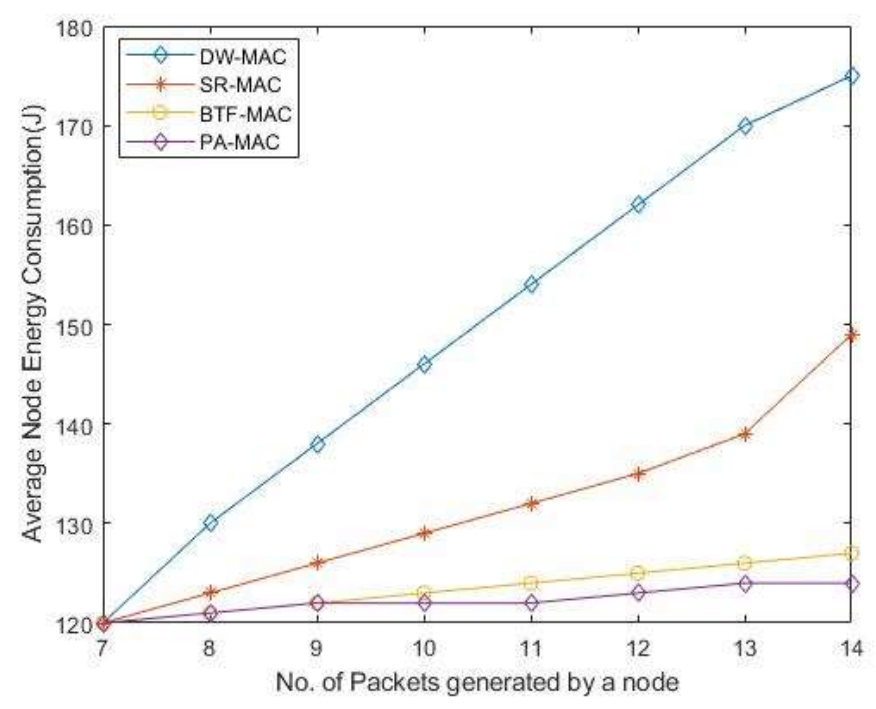

Figure 7. Average Node Energy Consumption versus No. of Packets

A comparative analysis of average node energy consumption with number of data packet generated from node is shown in the Figure 7. As shown in the figure, the PAMAC also outperforms other related protocols in average energy consumption. For example, under heavy traffic, in PA-MAC a node consumes energy less by more than $27 \%$ on average.

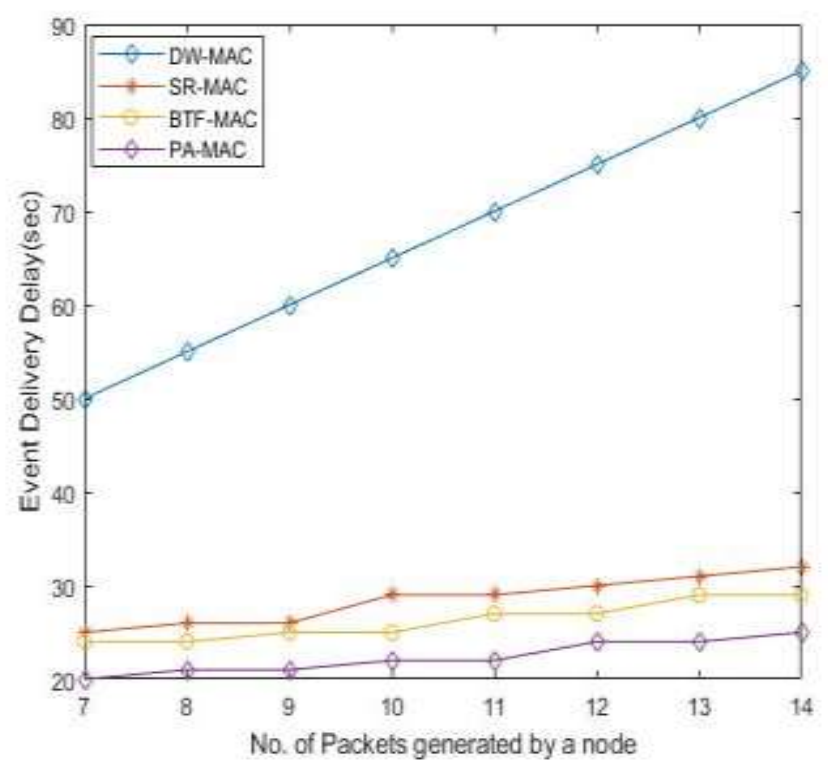

Figure 8. Average Event Delivery Delay versus No. of Packets

Figure 8 shows the event delivery delay of the protocols according to the number of data packets generated by a node Because DW-MAC cannot transmit multiple data packets in a single operational cycle, the event delivery delay in DW-MAC is the highest. According to the simulations, the PA-MAC outperforms other protocols due to the usage of priority period. 


\section{Conclusions}

In this paper, the authors present a Priority and Delay Aware packet transmission MAC Protocol for some applications such as field monitoring in which an abnormal data and normal data may be generated in WSNs. The proposed MAC protocol, called as PA-MAC, has a duty cycling scheme to support priority and reduce packet transmission delay to the final destination In PA-MAC protocol, one operation period is divided into 4 parts: Sync1 period, Data period, Sync2 period, and Sleep period. Other periods, except Sync2 period, work like those of other protocols such as RMAC, DW-MAC, and SR-MAC. During the Sync2 period all nodes wake up again to get the slot reservation and the operational cycle expansion information from a sink node. In other words, the Sync2 period is used for operational cycle expansion when burst data are generated.PA-MAC divides the DATA period into data packet slots and the SLEEP period into frames. And each frame in the SLEEP period is divided into sleep slots. Using priority bit field in SIF control frame, PA-MAC can support priority packet transmission first. A comparative analysis of PA-MAC and other related protocols such as DW-MAC, SR-MAC, and BTF-MAC was conducted through several simulations. PA-MAC outperforms these related protocols, with lower transmission delay, and node energy consumption especially for the priority data packet as number of packets from sensor nodes increases.

\section{Acknowledgments}

This research was supported by a 2018 Research Grant from Sangmyung University.

\section{References}

[1] W. Ye, J. Heidemann and D. Estrin, "An Energy-Efficient MAC Protocol for Wireless Sensor Networks", Proceedings of the 21st Annual Joint Conference of the IEEE Computer and Communications Societies, New York, NY, vol. 3, (2002), pp. 1567-1576.

[2] W. Ye, J. Heidemann and D. Estrin, "Medium access control with coordinated adaptive sleeping for wireless sensor networks", IEEE/ACM Transactions on Networking, vol. 12, no. 3, (2002), pp. 493-506.

[3] T. V. Dam and K. Langendoe, "An adaptive energy efficient MAC protocol for wireless sensor networks”, In Proc. the 1st Int. Conf. Embedded Networked Sensor Systems, (Sensys'03), Los Angeles, California, (2003) November 05-07, pp. 171-180

[4] W. Ye, F. Silva and J. Heidemann, "Ultra-Low Duty Cycle MAC with Scheduled Channel Polling", 4th ACM Conference on Embedded Networked Sensor Systems (SenSys'06), Bouder, Colorado, (2006) October 31 - November 03, pp. 321-334.

[5] J. Polastre, J. Hill and D. Culler, "Versatile low power media access for wireless sensor networks", In Proc. the 2nd Int. Conf. Embedded Networked Sensor Systems (Sensys'04), Baltimore, MD, (2004) November 03-05, pp. 95-107.

[6] A. El-Hoiydi and J. D. Decotignie, "WiseMAC: An Ultra Low power MAC protocol for multi-hop wireless sensor networks", the Proc. of the First International Workshop on Algorithmic Aspects of Wireless Sensor Networks (ALGOSENSORS 2004), Turku, Finland, LNCS 3121, (2004) July 16, pp. 18-31.

[7] M. Buettner, G. V. Yee, E. Anderson and R. Han, "X-MAC: A short preamble MAC protocol for dutycycled wireless sensor networks", In Proc. the 4th International Conference on Embedded Networked Sensor Systems, Boulder, Colorado, (2006) October 31-November 3, pp. 307-320.

[8] X. Wang, X. Wang, L. Liu and G. Xing, "DutyCon: a dynamic duty-cycle control approach to end-toend delay guarantees in wireless sensor networks", ACM Transactions on Sensor Networks, vol. 9, no. 4, (2013), pp. 1-42.

[9] Y. M. Mao, F. L. Kan and D. W. Zhang, "Research on MAC Protocol for Wireless Sensor Network", Advanced Materials Research, vol. 945-949, (2014), pp. 2368-2371.

[10] S. Du, A. Saha and D. Johnson, "RMAC: a routing-enhanced duty-cycle MAC protocol for wireless sensor networks", Proceedings of the 26th Annual IEEE Conference on Computer Communications (INFOCOM 2007) Barcelona, Spain, (2007) May 6-12, pp. 1478-1486.

[11] Y. Sun, S. Du, O. Gurewitz and D. Johnson, "DW-MAC: a low latency, energy efficient demand wakeup MAC protocol for wireless sensor networks", Proceedings of the Ninth ACM International Symposium on Mobile Ad Hoc Networking and Computing (MobiHoc'08), Hong Kong, Hong Kong, (2008) May 26-31, pp. 53-62.

[12] H. Tang, J. Cao, X. Liu and C. Sun, "SR-MAC: A Low Latency MAC Protocol for Multi-Packet Transmission in Wireless Sensor Networks", Journal of Computer Science and Technology (2013), vol. 28, no. 2, pp. 329-342. 
[13] H. Y. Kim, S. C. Kim, J. H. Jeon and J. J. Kim, "Traffic Adaptive Wakeup Control Mechanism in Wireless Sensor Networks", Journal of Korea Multimedia Society, vol. 17, no. 6, (2014), pp. 681-686.

[14] H. Y. Kim and S. C. Kim, "A Delay Efficient and Burst Traffics Friendly MAC Protocol in Wireless Sensor Networks", Journal of Korea Multimedia Society, vol. 20, no. 2, (2017), pp. 254-260.

[15] J. Y. Lee, S. C. Kim, "A Bursty Traffics Friendly MAC Protocol in Wireless Sensor Networks", Journal of the Korea Institute of Information and Communication Engineering, vol. 22, no. 5, (2018), pp. 772778.

[16] H. Y. Kim, S. C. Kim and H. J. Park, "Priority and Delay Aware packet transmission MAC Protocol for Wireless Sensor Network", International Journal of Security Technology for Smart Device, vol. 5, no. 2, (2018), pp. 9-14. 